\title{
Correction to: Keeling's Fetal and Neonatal Pathology
}

T. Yee Khong and Roger D.G. Malcomson

\section{Correction to:}

Chapter 2 in: T.Y. Khong, R.D.G. Malcomson (eds.), Keeling's Fetal and Neonatal Pathology, DOI 10.1007/978-3-319-19207-9

We received following corrections from author after publication of this book.

In table 2.9 in the 'Heart' column, the weights from 17 weeks onwards are incorrect. Now the revised version of table 2.9 is updated in chapter 2 . 
